\section{Highlights of this issue}

By Sukhwinder S. Shergill

\section{PTSD: frequency, violence and early treatment}

The shooting that occurred on Utøya Island, Norway in 2011 received worldwide coverage. This tragedy resulted from a terrorist - whose sanity was subsequently examined during his trial - killing 69 young people and leaving 56 severely injured. Dyb and colleagues (pp. 361-367) invited the 490 survivors of the attack to interview, and found that $11 \%$ fulfilled full criteria for post-traumatic stress disorder (PTSD) and another 26\% for partial PTSD. These levels were six times higher than the general population rate. Risk factors associated with higher rates of PTSD included being female, non-Norwegian, having lost a close friend and currently experiencing pain. Higher levels of social support were associated with reduced rates of PTSD. Females reported greater levels of emotional and physiological reactions at the time of the event and the authors suggest that this may be the trigger for the higher rates of PTSD in females, as opposed to a difference in the appraisals of threat. They also found that high levels of social support may be protective and concluded that this should be emphasised in any information provided after such disasters. Alisic et al (pp. 335-340) reported a very similar 15\% rate of PTSD in their meta-analysis of studies examining the rates of PTSD in children and adolescents exposed to trauma. They also found higher rates of PTSD not only in females, but also in children exposed to interpersonal trauma. A thoughtful editorial by Bisson (pp. 329-330) reviews the literature on the optimal post-trauma response to minimise PTSD. There is a need for evidence-based approaches, given the fact that single-session psychological debriefing may cause harm in some people, and both multiple-session psychosocial interventions and pharmacotherapy have limited proven benefit. He concludes that pragmatic support provided in an empathic manner is an optimal initial response, with a focus on the subsequent early detection of emergent PTSD and treatment with evidence-based approaches such as cognitive-behavioural therapy, selective serotonin reuptake inhibitors and eye movement desensitisation and reprocessing. In combination with alcohol misuse, PTSD was associated with a significantly increased risk of severe violence in community settings in US veterans having served in Iraq and Afghanistan. In the absence of alcohol misuse, PTSD showed a much weaker link with such violence; Elbogen and colleagues (pp. 368-375) suggest this interaction needs to be reflected in risk reduction plans for veterans.

\section{Stigma and disclosure, self-harm and problem-solving, and 25 years of Prozac}

There is toxic relationship between the stigma of mental illness evident in the general population and the impact this has on people with mental health difficulties. This negative public attitude can lead to self-stigmatisation, with detrimental effects on social functioning, loss of social support and increased levels of stress associated with maintaining secrecy. Rüsch et al (pp. 391-397) tested the effects of a Coming Out Proud peer-led group intervention and found no significant beneficial effect of the intervention on measures of self-stigma or empowerment. However, there were positive effects on measures of cognitive appraisal of stigma being a stressor, disclosure-related distress and benefits of disclosure of mental illness. Self-harm is an important risk factor for suicide, and problem-solving ability may mediate the relationship between stressors and self-harm behaviour. McAuliffe and colleagues (pp. 383-390) report that a structured group problem-solving skills programme did not demonstrate any additional benefit in the frequency of subsequent self-harm episodes, compared with treatment as usual. The group treatment also did not demonstrate any positive effects on psychological or social functioning, or on levels of depressive, impulsive or anxiety-related symptoms. The 25th birthday of Prozac is the inspiration for a review of the background to its highly successful launch, and suggests some lessons to be learnt for medical practice and drug development in psychiatry. Somewhat controversially, Shorter (pp. 331-332) suggests that, rather than offering a major advance in the treatment of depression, the success of Prozac was largely a lesson in successful marketing, with a little added popular science to catch the public's attention. The conclusion is that in both diagnosis and treatment: don't be fooled by the emperor's new clothes.

\section{Psychosis: cannabis, stress and mindfulness}

Stress has been linked as an aetiological factor in many psychiatric disorders for some time, but there is increasing interest in the interaction between environmental stressors and genetic predisposition to mental illness. Cullen and colleagues (pp. 354-360) report that children with a family history of schizophrenia, and children with epidemiological risk factors for schizophrenia, not only experienced more frequent negative life events but experienced greater distress consequent on them relative to their peers. The authors propose that there may be a difficulty in poor adaptive coping mechanisms causing persistently higher levels of distress. In a similar vein, Morgan et al (pp. 346-353) demonstrated that childhood abuse, life events and cannabis use may combine synergistically to increase psychotic experiences within a population-based sample. They suggest that early adverse experiences may create a vulnerability to psychosis, on which other such stressors such as cannabis use can subsequently act. There is an increased popular awareness of mindfulness as counteracting many psychological stressors that can contribute to poorer mental and physical health. Chadwick (pp. 333-334) suggests that this practice can also usefully be used therapeutically in psychosis; his editorial reviews recent successful adaptations of mindfulness for patients with psychosis and makes a case for increasing its clinical use, although he advises that this should be carried forward in association with careful training and supervision. 